\title{
DEVELOPMENT AND EVALUATION OF NANOEMULSION AS A CARRIER FOR TOPICAL DELIVERY SYSTEM BY BOX-BEHNKEN DESIGN
}

\author{
PALLAVI M CHAUDHARI ${ }^{1 *}$, MADHAVI A KUCHEKAR ${ }^{2}$
}

${ }^{1}$ Department of Pharmaceutics, Dr. DY Patil College of Pharmacy, Akurdi, Maharashtra, India. ${ }^{2}$ Department of Quality Assurance Techniques, Dr. DY Patil College of Pharmacy, Akurdi, Maharashtra, India.Email: pallavic26@gmail.com

Received: 31 March 2018, Revised and Accepted: 03 May 2018

\section{ABSTRACT}

Objective: The aim of this study was to develop a nanoemulsion for topical delivery.

Methods: Topical nanoemulsion was prepared by homogenization method. Box-behnken design was utilized to study the effect of oil, surfactant and Co-surfactant, on droplet size, entrapment efficiency and drug release. Nabumetone a non-steroidal anti-inflammatory drug was incorporated in castor oil with Tween 80 and Polyethylene glycol 600 to form the nanoemulsion by homogenization method. The nanoemulsion was further subjected to different evaluation parameters and ex-vivo study. The crystalline nature of drug was confirmed by powder X-ray diffraction studies. Drug-excipient compatibility was confirmed by Fourier transform infrared (FTIR) spectroscopy, differential scanning calorimetry (DSC), respectively.

Results: The average globule size of nabumetone-containing nanoemulsion decreased with decrease in concentration of oil and surfactant. Nanoemulsion was evaluated by $\mathrm{pH}$, rheology, globule size, zeta potential, scanning electron microscopy, DSC, FTIR spectroscopy, and stability. In vitro drug release shows maximum $84.35 \%$ permeation rate through cellophane membrane and ex-vivo drug release shows $86.32 \%$ permeation rate through goat skin.

Conclusion: Thus, the nanoemulsion formulated showed good results regarding topical delivery.

Keywords: Nabumetone, Castor oil, Box-Behnken design, Globule size, Drug release.

(C) 2018The Authors. Published by Innovare Academic Sciences Pvt Ltd. This is an open access article under the CC BY license (http://creativecommons. org/licenses/by/4. 0/) DOI: http://dx.doi.org/10.22159/ajpcr.2018.v11i8.26359

\section{INTRODUCTION}

The term "nanoemulsion" refers to a thermodynamically stable, isotopically clear dispersion of two immiscible liquids, such as oil and water, stabilized by an interfacial film of surfactant molecules $[1,2]$. A nanoemulsion is considered to be a thermodynamically or kinetically stable liquid dispersion of an oil phase and a water phase, in combination with a surfactant. The dispersed phase typically comprises small particles or droplets, with a size range of $50 \mathrm{~nm}-500 \mathrm{~nm}$, and has very low oil/water interfacial tension. Because the droplet size is $<25 \%$ of the wavelength of visible light, nanoemulsions are transparent. The nanoemulsion is formed readily and sometimes spontaneously, generally without high-energy input. In many cases, a cosurfactant or cosolvent is used in addition to the surfactant, oil phase, and the water phase [3].

Nabumetone is used to reduce pain, swelling, and joint stiffness from arthritis. This medication is known as a nonsteroidal anti-inflammatory drug (NSAID). NSAIDs are one of the numerous drugs having the limitation of poor aqueous solubility and bioavailability. In addition, prominent gastrointestinal (GI) side effects such as ulceration and high first pass metabolism are the prime obstacles that led to commercial withdrawal of certain drugs of this class-like nabumetone. Nowadays trying to overcome GI side effects by the topical delivery of NSAID. Skin has been shown to be a suitable delivery route for drugs formulated topically during the past few years. NSAID promotes local analgesic as well as, topical application of cyclooxygenase inhibitors suppresses ultraviolet (UV) radiation-B mediated cutaneous inflammation. Therefore, application of nabumetone topically has another important role in inhibiting UV-mediated inflammation. Duration of action of nabumetone can be increased by improving its release patterns from formulation and systemic anti-inflammatory effects without major GI side effects. Nowadays, the nanoemulsions are the potential drug delivery system for oral and parenteral administration of drugs like NSAID. It was found that nanoemulsions could be a very good carrier for topical delivery of highly lipophilic drugs.

It was also found that excipient which is used to manufacture nanoemulsion helps to augment the solubilizing and permeation capacity. Because of the smaller droplets contained in nanoemulsion thereby facilitate close contact with the stratum corneum. That is why, the amount of encapsulated agent penetrating into the viable skin facilitates the drug transport by changing the vehicle/stratum corneum partition coefficient $[4,5]$.

\section{MATERIALS AND METHODS}

Materials

Nabumetone was gifted from Cipla Patalganga Pvt., Ltd., Mumbai, India. Castor oil was purchased from local market, polyethylene glycol (PEG); tween 80 was gifted from SD fine chemicals laboratory, Mumbai, India, and all other chemicals used were of analytical grade.

\section{Methods}

Emulsification study

Screening of surfactant

Surfactant selection was done on the basis of percentage of transparency (\%Transparency) and ease of emulsification. Briefly, $2 \mathrm{ml}$ of each surfactant was added to the selected $2 \mathrm{ml}$ of oil phase. The mixture was gently heated at $50^{\circ} \mathrm{C}$ homogenization of the components.

Each $1 \mathrm{ml}$ mixture was then diluted with distilled water in a stoppered conical flask. Ease of emulsification was judged by the number of flask inversion required to yield a homogenous emulsion. Emulsion is allowed to stand for $2 \mathrm{~h}$ and their \% transparency was evaluated by UV visible spectrophotometer using distilled water as a blank at $236 \mathrm{~nm}$ [6]. 
Screening of co-surfactant

Screening of cosurfactant was conducted on the basis of \% transparency and ease of emulsification. $1 \mathrm{ml}$ of each cosurfactant mixed with $2 \mathrm{ml}$ of selected surfactant and the $3 \mathrm{ml}$ of selected oil phase was added and evaluated in a similar fashion as described in the above screening of surfactant.

\section{Pseudoternary phase diagrams}

To determine the concentration of components for the existing range of the nanoemulsion, a pseudo ternary phase diagram was constructed using a water titration method at ambient temperature $25^{\circ} \mathrm{C}$ with the help of CHEMIX ternary diagram software. The surfactant and co-surfactant, Smix was mixed at different weight ratio 1:1, 1:2, and 2:1. For each phase diagram, the ratio of oil to the Smix was mixed as 1 : 9,2:8,3:7,4:6,5:5,6:4,7:3,8:2,9:1(\%w/w) respectively. Afterward water was added drop wise to each oil-Smix mixture under gentle agitation to identify nanoemulsion region until mixture became clear at certain point. The concentration of components was recorded to construct the pseudoternary phase diagram and then the contents of oil, surfactant, cosurfactant, and water appropriate weight ratio were selected based on these results. The scale up of the proportions is easy, as the system is thermodynamically stable [7-9].

\section{Formulation of final screening batches of nanoemulsion}

Nanoemulsions are prepared when interfacial tension at oil/water is kept at very low level. Interfacial layer is kept very much flexible and fluid concentration of surfactants should be high enough to give surfactant molecules to be stabilized the nanoemulsion at an extremely low interfacial tension. According to the nanoemulsion area in the phase diagrams, the nabumetone loaded nanoemulsion formulations were selected at mixing oil, surfactant, and cosurfactant together and adding precisely water drop-by-drop to these oily phases with magnetic stirring. After that, these batches were evaluated. On basis of result of evaluation, concentration range of oil, Smix and water was selected.

\section{Formulation optimization using Box-Behnken experimental design [10]}

Preparation of nabumetone-loaded nanoemulsion was through mixing the nabumetone, oil, surfactant, cosurfactant and addition of water, with drop by drop gentle stirring at room temperature. After complete dissolution, the nabumetone-loaded nanoemulsion was obtained. Box Behnken design is composed of factorial experiment, axial points, center point. Box-Behnken design has been successfully used to optimize the technology or production conditions for drug delivery systems such as nanoemulsion, micro emulsion, liposomes, and microsphere in recent years. Based on the preliminary experiments and our previous studies, three formulation parameters, the oil, surfactant, and co-surfactant ratio, were identified as key factors responsible for the properties of nanoemulsion. Considering the feasibility of nanoemulsion at the extreme values, the ranges of three factors were determined as follows 1. Oil in $\mathrm{ml}$

2. Surfactant in $\mathrm{ml}$

3. Cosurfactant in $\mathrm{ml}$

Droplet size $\left(\mathrm{Y}_{1}\right), \%$ drug entrapment efficiency $\left(\mathrm{Y}_{2}\right)$ and \% drug release $\left(\mathrm{Y}_{3}\right)$ were selected as the responses because they are generally considered as significant factors for assessing the qualities of nanoemulsion and oil concentration $\left(\mathrm{X}_{1}\right)$, surfactant concentration $\left(\mathrm{X}_{2}\right)$, co surfactant concentration $\left(\mathrm{X}_{3}\right)$ were selected as independent variables [11].

Box-Behnken statistical design with three factors and 17 runs was selected to statistically optimize the formulation parameters and evaluate the main, interaction, and quadratic effects of the formulation ingredients on the droplet size, drug entrapment efficiency, drug release. 3-factor design was used to explore the quadratic response surfaces and for constructing contour plot thus helping in optimizing a process using a small number of experimental runs.
Statistical analysis of the Box-Behnken design batches were performed by multiple regression analysis using Design expert version 10 software. The contribution of each factor with different levels to the response was evaluated with two-way analysis of variance. The models were evaluated in terms of statistically significant regression coefficients $\left(\mathrm{R}^{2}\right)$ values.

\section{Characterization of nanoemulsions}

\section{Ease of emulsification and phase separation study}

Formulation $50 \mathrm{mg}$ was accurately weighed and diluted to $50 \mathrm{ml}$ with distilled water to yield fine emulsion. The ease of formation of emulsions was noted by noting the number of flask inversion required to give uniform emulsion. The resulting emulsions were observed visually for the relative turbidity. The emulsions were allowed to stand for $2 \mathrm{~h}$ and their transmittance was measured at $236 \mathrm{~nm}$ by UV visible spectrophotometer (Shimadzu UV-1700). Phase separation study was performed by added each $1 \mathrm{ml}$ nanoemulsion formulation to $10 \mathrm{ml}$ volumetric flask and diluted with distilled water up to the mark. After inverting volumetric flask for 3-4 times, each mixture was stored for $2 \mathrm{~h}$, and phase separation was observed visually.

\section{pH determination}

Each nanoemulsion formulation was checked using $\mathrm{pH}$ meter. The $\mathrm{pH}$ meter was calibrated before use in formulations using $\mathrm{pH} 4$ and $\mathrm{pH} 7$ standard buffer solutions. The $\mathrm{pH}$ meter electrode was immersed in $10 \%$ nanoemulsion and $\mathrm{pH}$ was recorded [12].

\section{Viscosity determination}

The viscosity was measured to determine rheological properties of formulations. Brookfield Rheometer viscometer (DV- $\Pi+$ version 10) at $30^{\circ} \mathrm{C}$ with a CPE 61 spindle at $100 \mathrm{rpm}$ was used to serve this purpose. Results were taken in triplicate, and the average was taken in to consideration.

\section{Globule size determination by digital microscope and Zetasizer}

$1 \mathrm{ml}$ emulsion was diluted to $1 \mathrm{ml}$ with distilled water. Ample was placed on glass slide and mean globule size of resulting emulsions determined by digital microscope (LABOMED microscopy).

The formulation $(0.1 \mathrm{ml})$ was dispersed in $50 \mathrm{ml}$ of water in a volumetric flask and gently mixed by inverting the flask. Measurement was done using a Zetasizer 1000 HS (Malvern Instrument, UK). Light scattering was monitored at $25^{\circ} \mathrm{C}$ at a $90^{\circ}$ angle [13].

\section{Zeta potential measurements}

The zeta potential of the diluted nanoemulsion formulae was determined using Zetasizer (Malvern Instruments, UK). Samples were placed in clear disposable zeta cells and results were recorded [14].

\section{Scanning electron microscopy (SEM)}

Morphology and structure of the nanoemulsion were studied using SEM. The sample was prepared by sprinkling the lyophilized solid lipid nanoparticle powder on a double adhesive tape, which was stuck on an aluminum stub. The sample was examined under a SEM (Joel JSM 6360) at an acceleration voltage of $10 \mathrm{kVA}$ [15].

\section{Interaction study by Fourier transform infrared (FTIR)}

FTIR spectra of prepared nanoemulsion were recorded on Shimadzu FTIR 8400 spectrophotometer. Sample was placed in sample holder; the scanning was performed between $4000 / \mathrm{cm}$ and $400 / \mathrm{cm}$ range [16].

\section{Differential scanning calorimetry (DSC)}

The nanoemulsion were hermetically sealed in aluminum pans and heated at a constant rate of $10^{\circ} \mathrm{C} / \mathrm{min}$ over temperature range of $40^{\circ} \mathrm{C}-300^{\circ} \mathrm{C}$. Inert atmosphere was maintained by purging nitrogen gas at flow rate of $50 \mathrm{ml} / \mathrm{min}$. 


\section{Drug entrapment efficiency}

Percentage drug entrapment efficiency was determined for drug content in formulations. The drug content of nabumetone nanoemulsion formulation was measured using UV visible spectroscopic method. Each $1 \mathrm{ml}$ sample was cooling centrifuged at $3500 \mathrm{rpm}$ for $30 \mathrm{~min}$. After centrifuge, supernatant transparent layer was taken and diluted with $10 \mathrm{ml}$ distilled water. The samples were measured as $236 \mathrm{~nm}$ using UV-VIS spectroscopic method. Results were taken in triplicate and the average was taken in to consideration [17].

\section{In-vitro drug release studies}

The in-vitro drug release studies were carried out using a Franz diffusion cell. The formulation was applied the surface of cellophane membrane which was placed between donor and receptor compartment of the Franz diffusion cell. Phosphate buffer pH 6.4 was used as dissolution media. The temperature of the cell was maintained at $37^{\circ} \mathrm{C}$ by circulating water jacket. This whole assembly was kept on a magnetic stirrer and the solution was stirred continuously using a magnetic bead.

$1 \mathrm{ml}$ of sample was spread over the cellophane membrane. Precautions were taken to ensure uniform nanoemulsion over the membrane and to remove all air bubbles between the nanoemulsion and the membrane. $2 \mathrm{ml}$ sample was withdrawn at suitable time intervals and dilute up to $10 \mathrm{ml}$ with same solvent and replaced with equal amounts of fresh dissolution media. Samples were analyzed spectrophotometrically at $236 \mathrm{~nm}$, and the cumulative \% drug release was calculated [18].

\section{Ex-vivo drug release study}

Franz diffusion cell was used in study for ex-vivo diffusion of drug. The cell consists of two chambers, the donor, and the receptor. The donor compartment is open at the top and is exposed to the atmosphere. The receptor compartment is surrounded by a water jacket for maintaining the temperature at $37^{\circ} \mathrm{C} \pm 2^{\circ} \mathrm{C}$ and is provided with a sampling port. The diffusion medium was $\mathrm{pH} 6.4$ phosphate buffer, which was stirred with magnetic beads (operated by a magnetic stirrer).

A goat skin as a membrane was placed between the two chambers. The diffusion media was stirred to prevent the formation of concentrated drug solution just beneath the membrane. Samples from the receptor compartment were taken at various intervals of time over a period of $6 \mathrm{~h}$ and the concentration of the drug was determined by UV spectrophotometric method using the standard curve.

Amount of drug diffused at various time intervals was calculated and plotted against time $[19,20]$.

\section{Thermodynamic stability tests}

Selected formulations were subjected to different thermodynamic stability tests. Only those formulations which survived dispersion stability tests were selected for further study [21].

\section{Heating cooling cycle}

All samples were placed between refrigerator temperature $4^{\circ} \mathrm{C}$ and $45^{\circ} \mathrm{C}$ of six cycles with storage at each temperature of not $<48 \mathrm{~h}$ was studied. Those formulations, which were stable at these temperatures, were subjected to centrifugation.

\section{Centrifugation}

Those formulations that passed were centrifuged at $3500 \mathrm{rpm}$ for 30 min using centrifuge. The formulations that did not show any phase separation were taken for further tests.

\section{Freeze thaw cycle}

Those samples which passed centrifugation test that placed between $-21^{\circ} \mathrm{C}$ and $+25^{\circ} \mathrm{C}$ three freeze thaw cycles with storage at each temperature for not $<48 \mathrm{~h}$ was done for the formulations, which passed these thermodynamic stress tests.

\section{RESULT AND DISCUSSION}

\section{Emulsification study}

\section{Screening of surfactants for emulsification ability}

From the solubility study, we selected oils and three surfactants for further study for selection of surfactants, it is necessary to have good emulsification ability along with good drug solubility. For this reason, selected three surfactants were screened for their emulsification ability with nabumetone. In emulsification study of surfactants, four combinations were evaluated for ease of emulsification and percent transmittance. After the screening for emulsification study, Tween 80 (surfactant) showed maximum transmittance hence selected for further study.

\section{Screening of cosurfactants for emulsification ability}

From preliminary screening of surfactants castor oil was selected as oil and Tween 80 was selected as surfactant for development of nanoemulsion formulation. In preliminary screening of co-surfactants, three co-surfactants were selected on the basis of previously done solubility study. In emulsification study of co-surfactants, three combinations were evaluated for ease of emulsification by flask inversion method and percent transmittance was measured.

Among different combinations castor oil (oil), Tween 80 (surfactant) and PEG 600 (Co-surfactant) required only 4-5 flask inversion and resultant emulsion showed maximum transmittance (94.7\%).

\section{Construction of pseudoternary phase diagrams}

A series of nanoemulsion were prepared and their nanoemulsifying properties were observed visually. The phase behaviors of castor oil and (Smix) showed. It was found the best ratio 2:1 gave more nanoemulsion region.

\section{Formulation optimization using Box-Behnken design}

Nanoemulsions batches were prepared by homogenization techniques which required two immiscible phases' oil and aqueous phase with an emulsifier helped in the formation of an emulsion by reducing the interfacial tension. The oil phases of the emulsion were prepared by dissolving nabumetone in castor oil with PEG 600 and Tween 80. Aqueous phase was injected to the oily phase with continuous homogenization at $2500 \mathrm{rpm}$ for $6 \mathrm{~h}$.

Box-Behnken design was used to optimize oil percentage and surfactant to cosurfactant ratio so as to get optimum globule size and desired percent drug entrapment efficiency and percent drug release. From the study, it was observed that both oil percentage and surfactant/ cosurfactant ratio was having significant effect on globule size and percent drug entrapment efficiency and percent drug release.

Formulation no.10 was found to satisfactory regarding globule size, entrapment efficiency, and drug release. This optimized batch was subjected to further characterization studies.

\section{Analysis of data using Box-Behnken design \\ Effect on globule size}

The Model F-value of 135.11 implies the model is significant. $p<0.0500$ for model, model term, indicated model and study was significant. $\mathrm{R}^{2}$ value was significant (0.9983). Response surface graph of globule size is shown in Fig. 1. The effect of concentration of oil and surfactant on globule size of nanoemulsion was observed. The globule size of nanoemulsion increased as oil and surfactant concentration was increased.

Final equation in terms of coded factors:

Globule size $=+425.13+108.26 * \mathrm{~A}+34.27 * \mathrm{~B}+5.71 * \mathrm{C}-{ }^{*} \mathrm{AB}-$ $0.41 * \mathrm{AC}+2.17 * \mathrm{BC}+35.5^{*} \mathrm{~A}^{2}-12.53^{*} \mathrm{~B}^{2}-16.59^{*} \mathrm{C}^{2}$ 
Effect on entrapment efficiency

The model F-value of 232.92 implies the model is significant value $<0.0500$ for model, model term, indicate model and study was significant. $\mathrm{R}^{2}$ value was significant (0.9967). Response surface graph of percent entrapment efficiency is shown in Fig. 2. The effect of concentration of oil and surfactant on entrapment efficiency (\%) of nanoemulsion was observed. The drug entrapment efficiency of nanoemulsion was increased as oil and surfactant concentration was decreased.

Final equation in terms of coded factors:

$+78.27+6.82 * \mathrm{~A}+3.51^{*} \mathrm{~B}+1.65^{*} \mathrm{C}-0.025^{*} \mathrm{AB} *-1.10^{*} \mathrm{AC}-1.89^{*} \mathrm{BC}-0.51^{*} \mathrm{~A}^{2}-$ $0.88 * B^{2}-0.99 * C^{2}$

Effect on drug release (\%)

The model F-value of 220.99 implies the model is significant value $<0.0500$ for model, model term, indicate model, and study was significant. $\mathrm{R}^{2}$ value was significant $(0.9965)$. Response surface graph of percent cumulative drug release is shown in Fig. 3. The effect of concentration of oil and surfactant on drug release (\%) of nanoemulsion was observed. The drug release (\%) of nanoemulsion was increased as oil and surfactant concentration was decreased.

Final equation regarding coded factors:

$+75.37+7.42 * \mathrm{~A}+2.47 * \mathrm{~B}+1.13^{*} \mathrm{C}-0.43^{*} \mathrm{AB}^{*}-0.63^{*} \mathrm{AC}-0.048^{*} \mathrm{BC}-$ $1.13 * \mathrm{~A}^{2}+0.022 * \mathrm{~B}^{2}-0.25 * \mathrm{C}^{2}$

All graphs showed that the relation of concentration of surfactant, concentration of co-surfactant, and oil on drug release. The positive sign indicate positive effect and negative sign indicate negative effect.

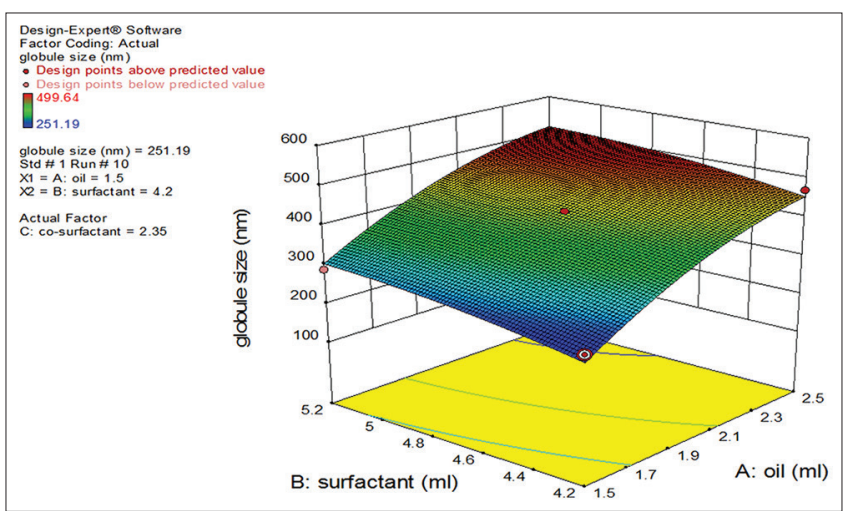

Fig. 1: Three-dimensional response surface curves for globule size

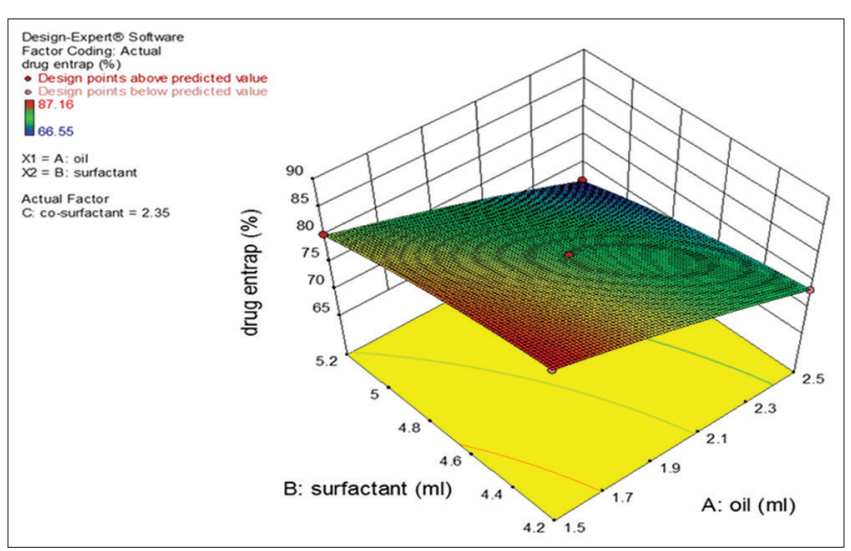

Fig. 2: Three-dimensional response surface curves for entrapment efficiency
Concentration of surfactant, concentration of co-surfactant, and oil showed that it has direct relation with drug release.

Effect of oil on globule size, entrapment efficiency, and drug release The globule size was decreased with decreased oil concentration nanoemulsion. The small droplet size gives them inherent stability against creaming, sedimentation, flocculation, and coalescence. It also allows the effective transport of active ingredients to the skin. The drug entrapment efficiency was increased with decreased oil concentration. Lower concentration of oil was decreased flowing rate there for increased drug entrapment efficiency. If increased oil concentration in nanoemulsion then decreased drug release. With increase in drug release was observed, with decreased viscosity and oil concentration.

Effect of surfactant on globule size, entrapment efficiency, and drug release

Globule size decreased with surfactant concentration decreased. Surfactant reduced the surface tension of oil drops, which help for breakdown of the oil drops into smaller size. Enough surfactant was present to cover the oil droplets surface which stabilized and prevented the coalescence of nanoemulsion droplets. Low surfactant concentration was to produce stable nanoemulsion. Tween 80 has higher HLB value that helps to increase the solubility of drug in oil and surfactant also increased the entrapment efficiency and drug release.

\section{Evaluation of nanoemulsion}

Ease of emulsification and phase separation study

Transmittance study revealed that as the concentration of surfactant increases the transmittance of resulting emulsions increases. The

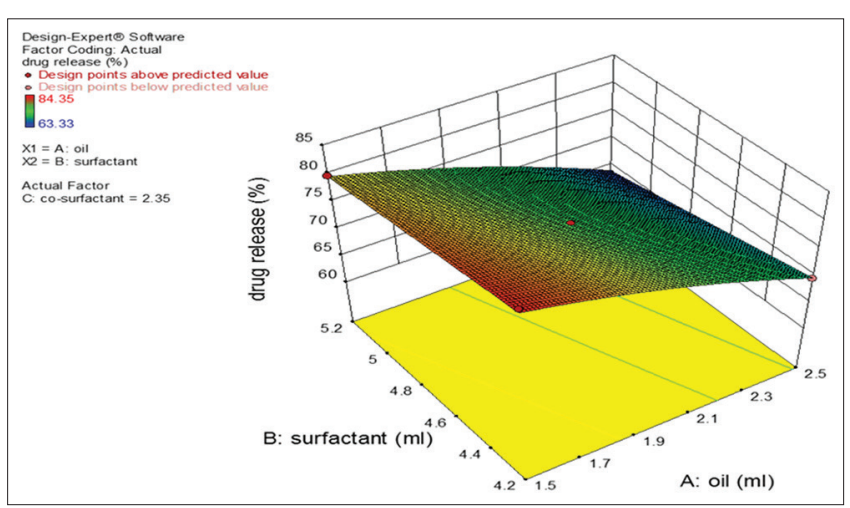

Fig. 3: Three-dimensional response surface curves for percent drug release

Table 1: Ease of emulsification of formulations

\begin{tabular}{llll}
\hline $\begin{array}{l}\text { Formulation } \\
\text { code }\end{array}$ & $\begin{array}{l}\text { No. of flask } \\
\text { inversions }\end{array}$ & \% transmittance & $\begin{array}{l}\text { Phase } \\
\text { separation }\end{array}$ \\
\hline $\mathrm{F}_{1}$ & 5 & 86.20 & No \\
$\mathrm{F}_{2}$ & 9 & 87.91 & No \\
$\mathrm{F}_{3}$ & 6 & 85.50 & No \\
$\mathrm{F}_{4}$ & 4 & 87.20 & No \\
$\mathrm{F}_{5}$ & 5 & 82.50 & No \\
$\mathrm{F}_{6}$ & 6 & 84.20 & No \\
$\mathrm{F}_{7}$ & 7 & 79.20 & No \\
$\mathrm{F}_{8}$ & 8 & 68.15 & No \\
$\mathrm{F}_{9}$ & 7 & 82.80 & No \\
$\mathrm{F}_{10}$ & 5 & 97.53 & No \\
$\mathrm{F}_{11}$ & 9 & 90.10 & No \\
$\mathrm{F}_{12}$ & 7 & 77.70 & No \\
$\mathrm{F}_{13}$ & 5 & 83.44 & No \\
$\mathrm{F}_{14}$ & 8 & 80.10 & No \\
$\mathrm{F}_{15}$ & 6 & 86.46 & No \\
$\mathrm{F}_{16}$ & 7 & 91.39 & No \\
$\mathrm{F}_{17}$ & 7 & 93.64 & No \\
\hline
\end{tabular}


ease of emulsification or rate of emulsion formation was measured by UV-spectrophotometer. All formulations were stable. No phase separation occurred for any formulation. Hence, all the formulations were subjected for further evaluation which mention in Table 1.

\section{pH determination}

$\mathrm{pH}$ of formulations which are applied topically must not be too high or low as it leads to skin irritation. $\mathrm{pH}$ of formulation was found to be in range of 5-6, which are very close to skin pH Fig. 4.

\section{Viscosity measurement}

The viscosity of formulations depends on the amount of surfactant cosurfactant mixture is shown in Fig. 5. The low viscosity of formulation F10 was assumed to be due to the presence due to low amount of Tween 80 and large amount of water

\section{Globule size determination}

By digital microscope

The globule size of the nanoemulsions also determines the rate and extent of drug release. The smaller the globule size, larger the surface area provided for drug absorption. Globule sizes of all formulations were observed $251.19 \mathrm{~nm}-488.64 \mathrm{~nm}$ determined by microscopy shown in Fig. 6. Among the batches, $F_{10}$ formulation showed globule

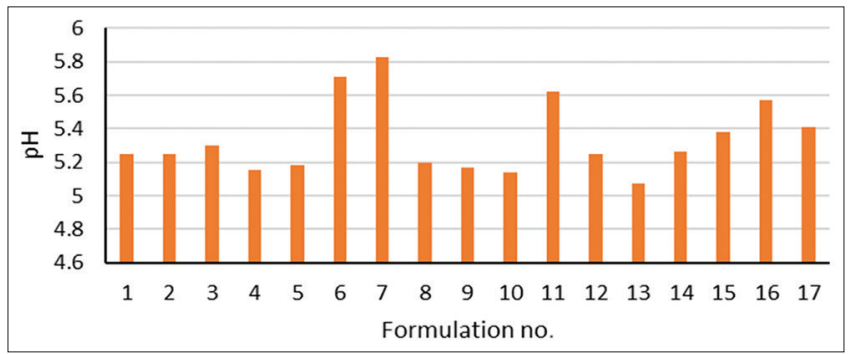

Fig.4: Histogram representation of $\mathrm{pH}$ of nabumetone nanoemulsion formulations

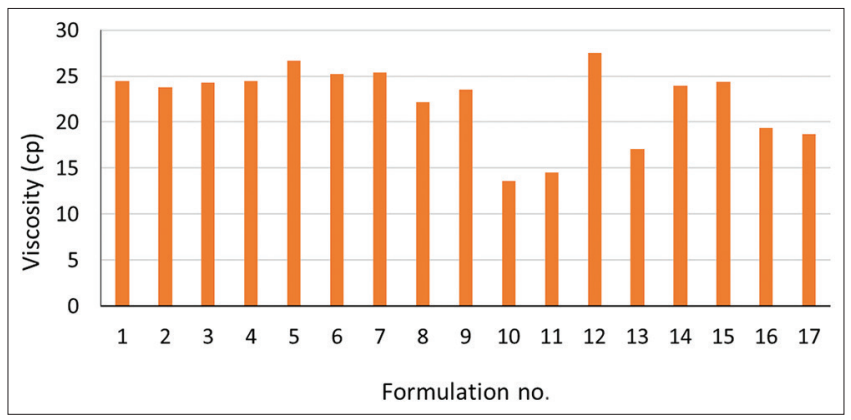

Fig.5: Histogram representation the of viscosity of nabumetone nanoemulsion formulations
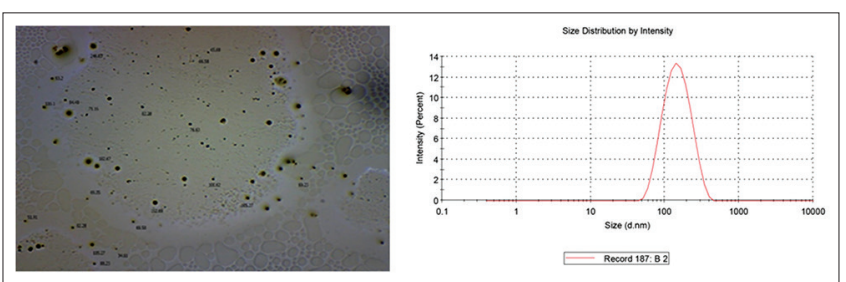

Fig. 6: Digital microscopy micrographs of optimized nabumetone nanoemulsion formulation F10 and by zetasizer size was found to be $251.19 \mathrm{~nm}$.

\section{By zetasizer 1000 HS}

The globule size of optimized nanoemulsion peak is shown at particle size $129.3 \mathrm{~nm}$ shown in Fig. 6. The graph depict that it has a homogeneous distribution of particles. Thus, the result showed that the particle size of formed nanoemulsion was in the required range, therefore, a transparent nanoemulsion formulated successfully.

\section{SEM}

SEM of nabumetone-loaded nanoemulsion is shown Fig. 7. It was observed that the nanoemulsion were uniform, spherical in shape.

\section{Zeta potential measurements}

Zeta potential of nanoemulsion formulation was determined to observe net surface charge on Globules and surrounding system. The magnitude of the zeta potential gives the indication of the potential stability of the colloidal system. If nanoemulsion has a negative sign indicate $\mathrm{pH}$ of 7.4 and $\mathrm{pH} 3$ indicate positive charge, they will tend to repel each other and there will be no tendency for the particles to come together. Zeta potential of optimized nanoemulsion formulation was at -15.26 $\mathrm{mv}$ shown in Fig. 8. Zeta potential in the range to $-30 \mathrm{mv}$ to $+30 \mathrm{mv}$ is common for stabilized nanoemulsion, thus the zeta potential value indicated that the electrostatic repulsion between particles will prevent their aggregation and thereby stabilize the nano particulate dispersion.

\section{FTIR study of nanoemulsion}

The FTIR spectrum of nanoemulsion is shown in Fig. 9. In the spectra, it

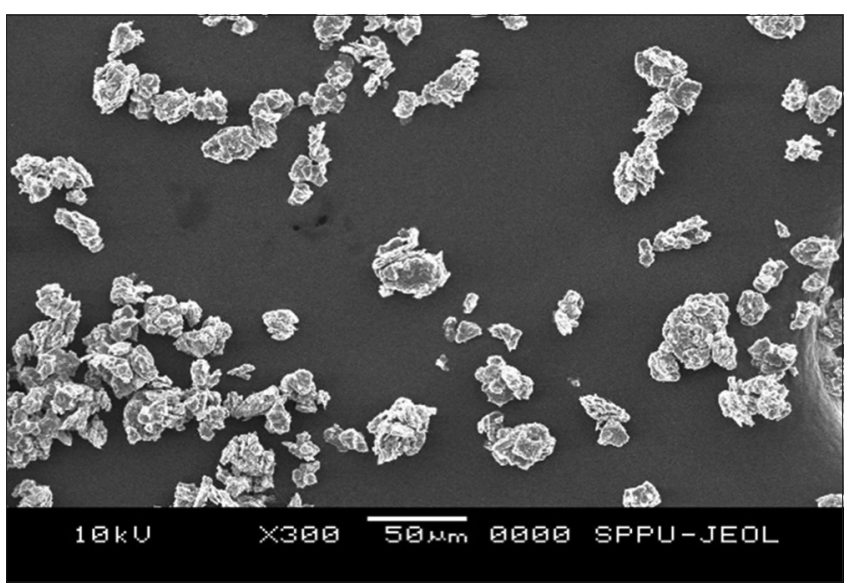

Fig. 7: Scanning electron microscopy micrograph of optimized nanoemulsion formulation F10

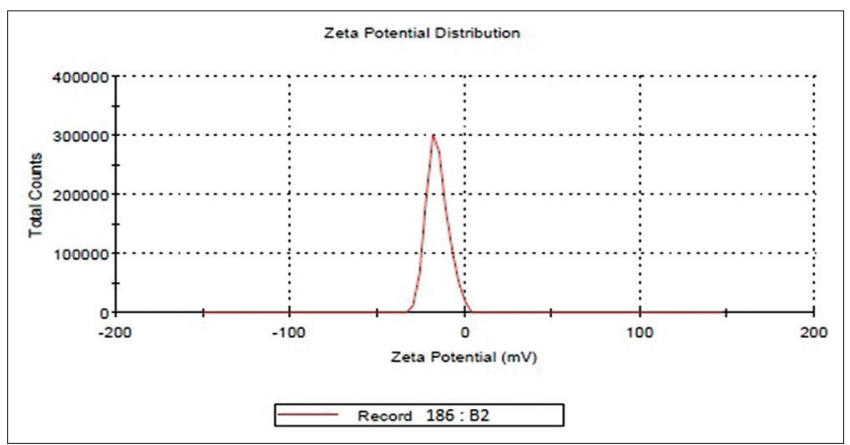

Fig. 8: Zeta potential micrograph of optimized nabumetone nanoemulsion formulation F10 
was observed that there was masking of some peak which is observed in drug spectra. It indicates that proper entrapment of drug and formation of nanoemulsion.

DSC

There was a significant change found in DSC peak intensity of peak and melting point of formulation was increased which showed that the formation of nanoemulsion in Fig. 10.

\section{Drug entrapment efficiency}

The entrapment efficiency of all nanoemulsions was found in range of 67.71-87.16\%. All formulations showed good entrapment efficiency is shown in Fig. 11.

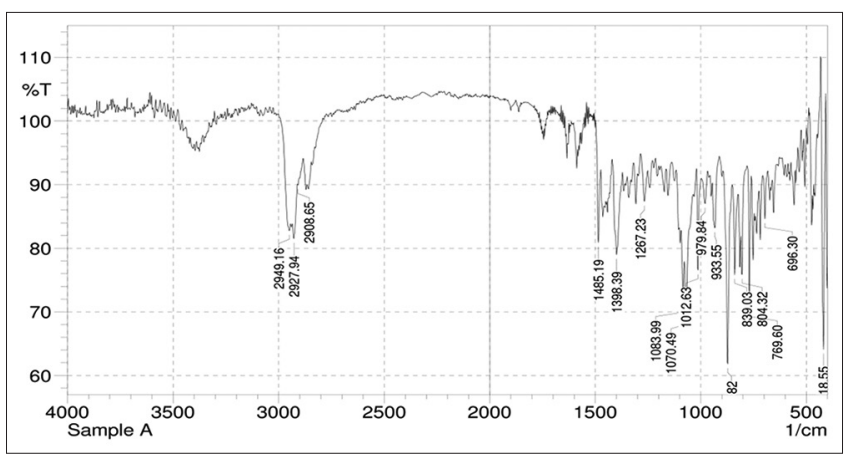

Fig. 9: Fourier transform infra-red spectroscopy image of optimized nabumetone nanoemulsion formulation F10
In-vitro drug release studies

The in-vivo release of nabumetone from the nanoemulsion was varied in amount according to concentration of emulsifying agents used in formulations. Drug release was found to be 65.33-84.35\% in Fig. 12. From the study, it was concluded that nanoemulsion showed better drug release within $7 \mathrm{~h}$.

\section{Ex-vivo study drug release study}

The drug release study was done using Franz's diffusion cell. The study was done for $7 \mathrm{~h}$ with an optimum interval of sampling. The goat skin was used as the diffusion membrane for the permeation studies. From the study of the release, it was observed that F10 showed percent cumulative drug of $86.32 \%$. That nanoemulsion drug release study as shown in Fig. 13.

\section{Stability study of nanoemulsion}

Nanoemulsions are considered to be thermodynamically stable systems which are formed at a particular concentration of oil, surfactant, and water, with no phase separation, creaming or cracking. Selected formulation from phase diagram were subjected to different stress stability testing such as heating cooling cycle, centrifugation, and freeze thaw cycle. All nanoemulsion formulations cycles were found to be stable.

\section{DISCUSSION}

In the coming years, topical drug delivery will be used extensively to impart better patient compliance. Since nanoemulsion is helpful in enhancing skin permeation, this novel drug delivery becomes popular. Moreover, they will become a solution for loading hydrophobic

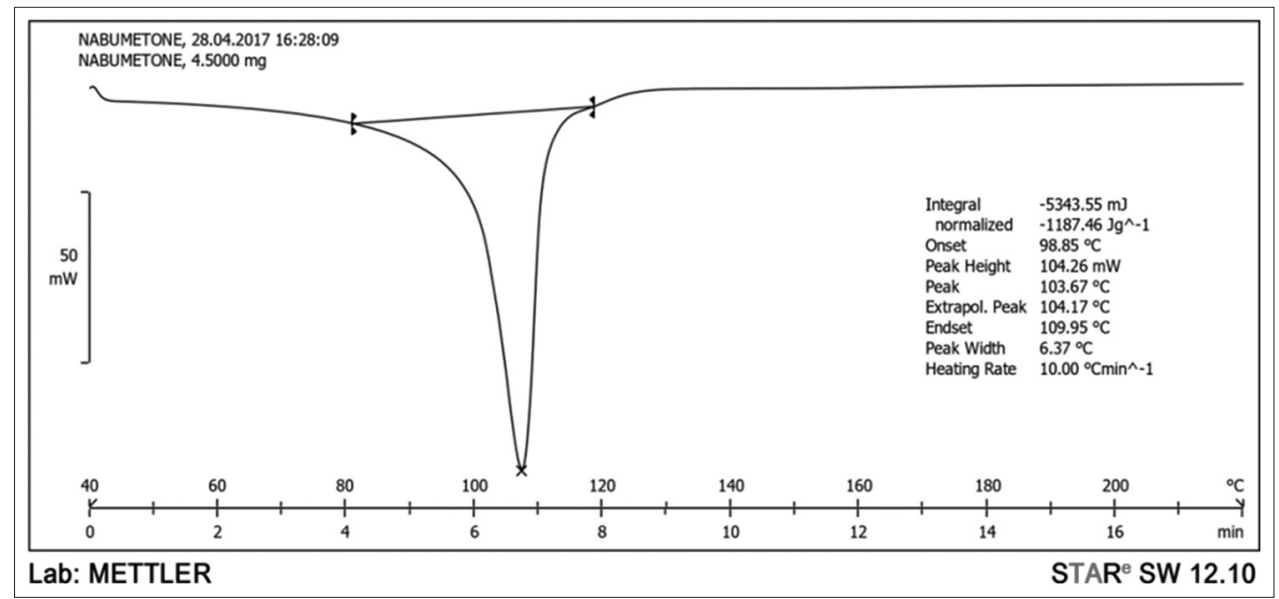

Fig. 10: Differential scanning calorimetry thermogram of optimized nabumetone nanoemulsion formulation F10

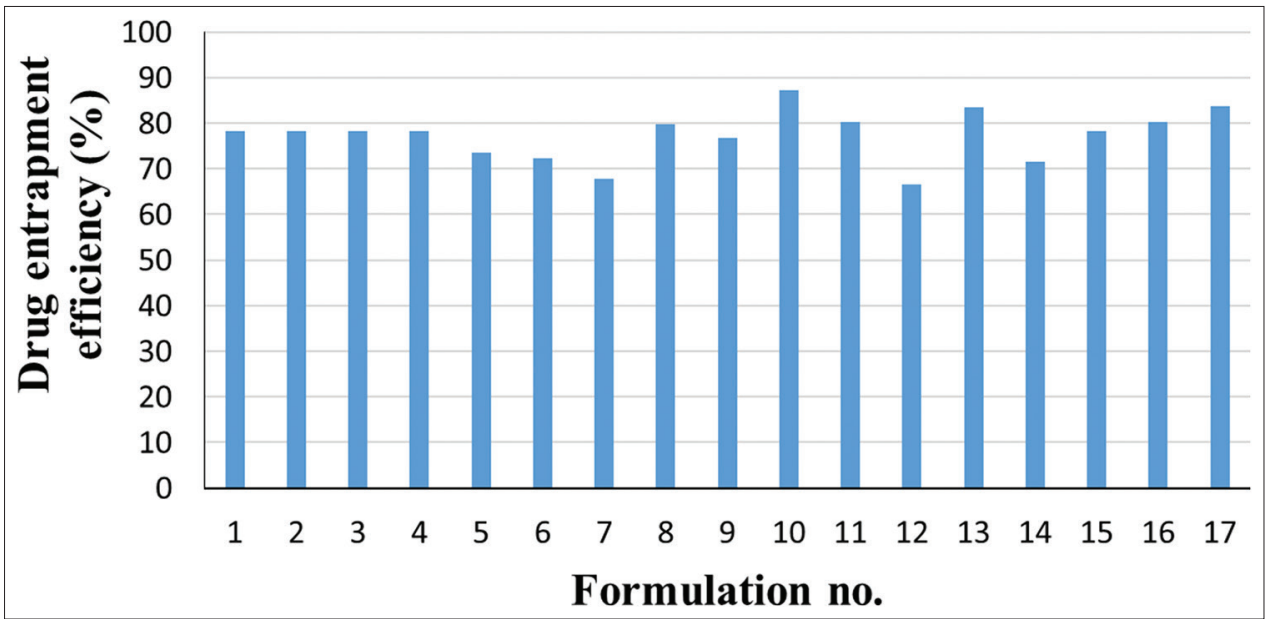

Fig. 11: Histogram representation of drug entrapment efficiency (\%) of all nabumetone nanoemulsion formulations 


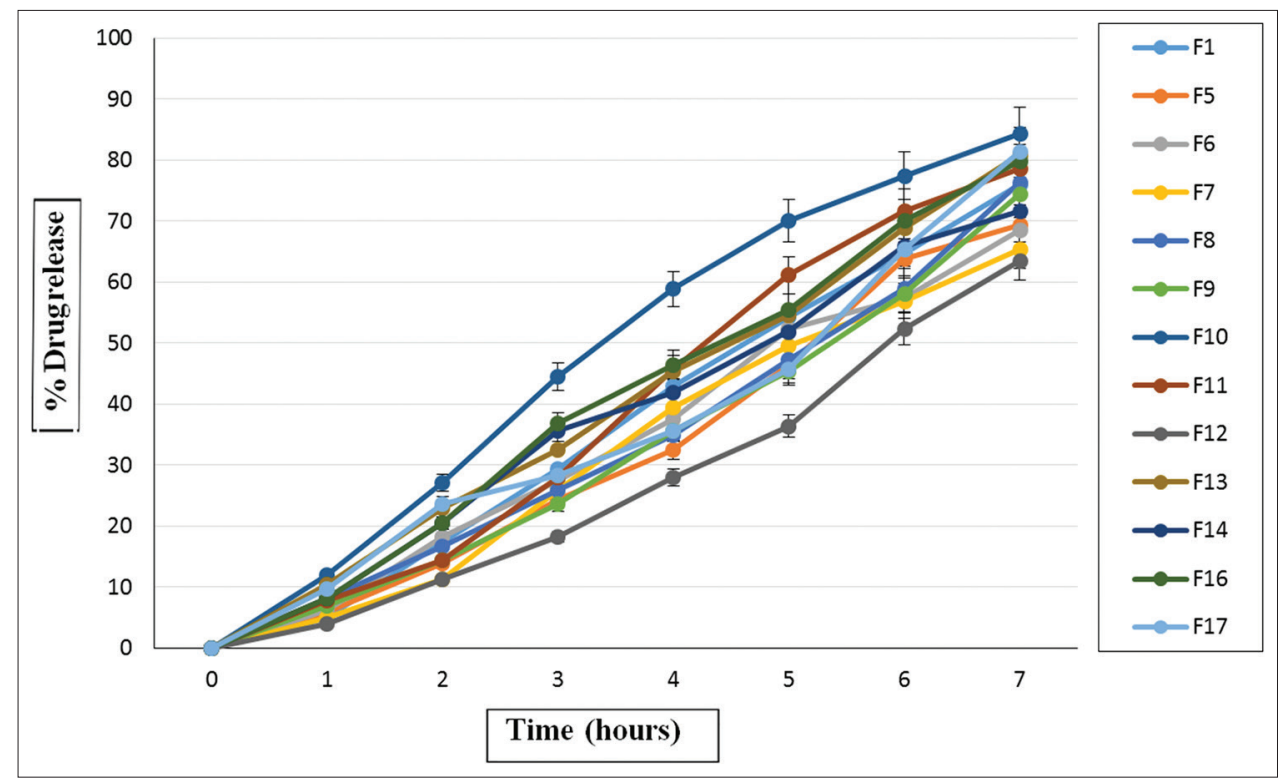

Fig. 12: In vitro release of nabumetone nanoemulsions (mean \pm standard deviation, $n=3$ )

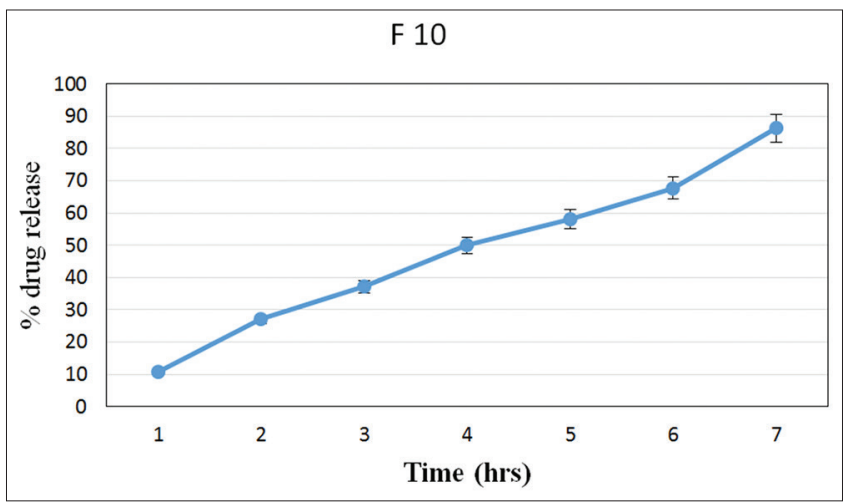

Fig. 13: Ex-vivo release of optimized nabumetone nanoemulsion F10 (mean \pm standard deviation, $n=3$ )

drugs in water soluble bases for the long-term stability. Similarly, in the study, topical nanoemulsion of nabumetone was formulated and subjected to physicochemical studies, i.e. rheological studies, $\mathrm{pH}$, and in-vitro release studies through cellophane membrane. The identification and preformulation study of nabumetone was carried out. DSC study of drug showed sharp peak of melting point which gave information about purity. FTIR study showed functional group present in nabumetone. X-ray powder diffraction study showed that high intensity of peak which indicates the crystalline nature of nabumetone.

Nanoemulsion prepared by homogenization technique, was thus used to formulate optimize formulation. F10, being the optimized formulation, was selected that contained castor oil $(1.5 \mathrm{ml})$, Tween $80(4.2 \mathrm{ml})$, PEG $600(2.35 \mathrm{ml})$, and stirring speed $(2500 \mathrm{rpm})$ gave desired nanometer particle size $(251.19 \mathrm{~nm})$, proper entrapment efficiency of drug (87.16\%) and drug release $(84.35 \%)$ after $7 \mathrm{~h}$. Response surface graph showed that concentration of oil and surfactant increased with increased particle size. Drug entrapment efficiency and percentage drug release were increased with decreased concentration of oil and surfactant. The present investigation has shown that it is possible to topical effect of nabumetone by nanoemulsion with castor oil, tween 80, and PEG 600 on topical delivery.

\section{CONCLUSION}

Optimization of castor oil, tween 80, and PEG 600 was used to form a stable nabumetone nanoemulsion formulation for topical delivery.

\section{ACKNOWLEDGMENT}

The authors acknowledge the Department of Physics, Savitribai Phule Pune University for providing facility for completion of research.

\section{AUTHOR'S CONTRIBUTION}

The authors state that no conflict of interest and initiated with the idea for preparation of the formulations and after the results obtained prepared this manuscript.

\section{CONFLICT OF INTERESTS}

Declared none.

\section{REFERENCES}

1. Sarker A, Shimu IJ, Haque TM, Asgher RA. Nanoemulsion: An excellent mode for delivery of poorly soluble drug through different routes. J Chem Pharm Res 2015;7:96676.

2. Jaiswal M, Dudhe R, Sharma PK. Nanoemulsion: An advanced mode of drug delivery system. Biotech 2015;5:123-7.

3. Lovelyn C, Attama AA. Current state of nanoemulsion in drug delivery. J Biomater Nanotechnol 2011;10:626-39.

4. More A, Ambekar AW. Development and characterization of nanoemulsion gel for topical drug delivery of nabumetone. Int J Pharm Pharm Res 2016;7:126-57.

5. Sugumar S, Mukherjee A, Chandrasekaran N. Nanoemulsion formation and characterization by spontaneous emulsification: Investigation of its antibacterial effects on listeria monocytogenes. Asian J Pharm 2015;23:23-8.

6. Modi JD, Patel JK. Enhancement of transdermal based gel delivery of aceclofenac using nanoemulsion as a vehicle. J Pharm Res 2012;5:214-8

7. Kavitha K, Kanagathara N. Optimization and solubilisation study of novel nanoemulsion formulation for 5-fluorouracil by applying pseudoternary phase diagram. Asian J Pharm and Clin Res 2014;7:137-9.

8. Singh BP, Kumar B, Jain SK, Shafaat K. Development and characterization of a nanoemulsion gel formulation for transdermal delivery of carvedilol. Int J Drug Dev Res 2012;4:151-61.

9. Aparna C, Srinivas P, Patnaik K. Enhanced transdermal permeability of telmisartan by a novel nanoemulsion gel. Int J Pharm Pharm Sci 2015;7:335-42. 
10. Sisinthy SP, Rao NK, Sarah CY. Design, optimization and in vitro characterization of self nano emulsifying drug delivery system of Olmesartan medoxomil. Int J Pharm Pharm Sci 2017;9:94-101.

11. Lala RR, Awari NG. Nanoemulsion-based gel formulations of COX2 inhibitors for enhanced efficacy in inflammatory conditions. Appl Nanosci 2014;4:143-51.

12. Malgope A, Murthy PN, Ramani R, Dey S. Development of nanoemulsion as carrier for transdermal delivery of valsartan. Int $\mathrm{J}$ Pharm Chem Sci 2013;2:1655-65.

13. Soheyla H, Foruhe Z. Effect of zeta potential on the properties of nano-drug delivery systems-a review (Part 1). Trop J Pharm Res 2013;12:255-64.

14. Rodriguez SG, Allemann E, Fessi H, Doelker E. Physicochemical parameters associated with nanoparticle formation in the salting-out, emulsification-diffusion, and nanoprecipitation methods. Pharm Res 2004;21:1428-39.

15. Patil SS, Mohite SK. Development and evaluation of solid dispersion incorporated topical gel of nabumetone. Res J Pharm Tech 2014;7:1413-9.
16. Patel MR, Patel MH, Patel RB. Preparation and in vitro/ex vivo evaluation of nanoemulsion for transnasal delivery of paliperidone. Appl Nanosci 2016;6:1095-1104.

17. Patel HC, Parmar GR, Seth AK, Patel JD, Patel SR. Formulation and evaluation of o/w nanoemulsion of ketoconazole. Am Int J Pharm Sci 2013;4:338-51.

18. Teixeira MC, Severino P, Andreani T, Boonme P, Santini A, Silva AM. D-a-tocopherol nanoemulsions: Size properties, rheological behavior, surface tension, osmolarity and cytotoxicity. Saudi Pharm J 2017;25:231-5.

19. Affandi MM, Julianto T, Majeed BA. Development and stability evaluation of astaxanthin nanoemulsion. Asian J Pharm Clin Res 2011;4:142-8

20. Borges VR, Simon A, Sena AR, Cabrel LM. Nanoemulsion containing dapsone for topical administration: A study of in vitro release and epidermal permeation. Int J Nanomed 2013;8:535-44.

21. Setya S, Talegaonkar S, Razdan BK. Nanoemulsions: Formulation methods and stability aspects. World J Pharm Pharm Sci 2014;3:2214-28. 